\title{
Conducta resiliente en persona mayor condenada por abuso sexual del centro penitenciario y carcelario INPEC de Girardot, Colombia: Sistematización de la práctica profesional en Trabajo Social
}

\author{
Resilient behavior in elderly person convicted \\ of sexual abuse, of the penitentiary and \\ prisoner's center INPEC of Girardot, Colombia: \\ Systematization of the professional practice in \\ Social Work
}

\section{Marisol Martínez Suarez* \\ María Otilia Pulecio Bazurto** \\ Eliana Marcela Acosta***}

\begin{abstract}
Resumen: La sistematización surge como una posibilidad para evaluar la experiencia vivida en el ejercicio de la práctica profesional con las personas mayores que se encuentran privadas de la libertad en el centro penitenciario y carcelario INPEC de la ciudad de Girardot. Dichas personas han sido condenadas por abuso sexual y se encuentran en un proceso de intervención orientado a la socialización y al fortalecimiento de conductas resilientes mediante una estrategia de formación pedagógica que explora las dimensiones corporales, afectivas, psicosociales y cognitivas de esta población. Para esto se usó el enfoque teórico-crítico propuesto por Cadena (1987, p. 41-52), quien sugiere un esquema para sistematizar experiencias organizativas, de capacitación y promoción (CIFUENTES, 1999, p. 46). Los resultados obtenidos permiten fortalecer los desarrollos de las líneas de intervención practicadas por los estudiantes de Trabajo Social y aplicadas en el centro penitenciario y carcelario de Girardot, garantizando la aceptación social de este grupo excluido por su conducta delictiva.
\end{abstract}

Palabras clave: intervención social, abuso sexual, persona mayor, conducta resiliente.

\footnotetext{
* Trabajadora Social, Magíster en intervención social y líder del semillero Desde las prácticas profesionales, reflexión de la intervención en Trabajo Social. Unidad académica Trabajo Social, UNIMINUTO, Sede Cundinamarca. marisol.martinez@ uniminuto.edu, maridesol13@hotmail.com

${ }^{* *}$ Trabajadora Social, Especialista en Gerencia de Proyectos, Magister en Salud Mental Comunitaria, Facultad de Enfermería de la Universidad El Bosque. Unidad Académica Trabajo Social, UNIMINUTO Sede Cundinamarca. maria.pulecio@uniminuto. edu, otip80@hotmail.com

${ }^{* * *}$ Trabajadora Social, UNIMINUTO, Centro Regional Girardot. eiriarteaco@uniminuto.edu.co
} 


\begin{abstract}
The systematization arises as the possibility of evaluating the experience lived in the exercise of professional practice with older people who are deprived of their liberty in the penitentiary and prison INPEC of the city of Girardot, condemned for sexual abuse, and they find them selves in an intervention process oriented towards the socialization and strengthening of resilient behaviors, based on a pedagogical training strategy that explores the corporal, affective, psychosocial and cognitive dimensions of the population. The critical theoretical approach proposed by (CADENA, 1987, p .41-52) was used, in which it suggests a scheme to systematize organizational, training and promotion experiences (CIFUENTES, 1999, p. 46). The obtained results allow to strengthen the processes developed by the lines of intervention practiced by students of Social Work and applied in the penitentiary center of Girardot, guaranteeing the social acceptance of this group, excluded for their criminal behavior.
\end{abstract}

Keywors: Social intervention, Sexual abuse, Elderly person, Resilient behavior.

Recebido em: 11/07/2019. Aceito em: 28/11/2019

\title{
Introducción
}

La intervención profesional con la población privada de la libertad perteneciente al grupo de persona mayor del Establecimiento Penitenciario de Mediana Seguridad (EPMS) se propone fortalecer la conducta resiliente. Esta es entendida como la capacidad de una persona para superar circunstancias traumáticas, concibiéndola como un asunto de reconocimiento, de generación de capacidades y acciones para fomentar capacidades de adaptación en el sujeto. Lo anterior se posibilita bajo la creación, el diseño y la implementación de una serie de estrategias pedagógicas que incluyen temáticas relacionadas con el proceso para alcanzar la resiliencia y cercarlos elementos que han deteriorado la calidad de vida de las personas privadas de la libertad.

Esto se puede prevenir con actividades que exploran las dimensiones corporales, afectivas, psicosociales y cognitivas de esta población, procurando una comprensión mayor sobre la importancia de la implementación de estos hábitos para su diario vivir dentro del establecimiento. De esta forma, se fomenta el fortalecimiento y reconocimiento de conductas resilientes con la población atendida a partir de la propia comprensión de sus realidades, necesidades y potenciales como individuos y como grupo. Lo anterior se hace teniendo en cuenta el delito cometido (abuso sexual) y la exclusión que reciben por parte de los demás internos y, en general, por parte de la población del instituto penitenciario y carcelario en mención.

Desde el enfoque crítico dialéctico propuesto se favorece la realización de diversos ejercicios de acercamiento a las personas mayores del centro penitenciario y la aplicación de instrumentos propios de la sistematización: la entrevista estructurada, el sociodrama (la colcha de retazos), los relatos de vida, los juegos de fotos y la ficha de recuperación de la experiencia. Con estos instrumentos es posible observar que la educación social presenta un cambio positivo en el diseño de estrategias personales y colectivas para apropiar el concepto y la conducta resiliente, tomando 
como punto de partida las oportunidades generadas por el equipo de trabajadoras sociales que realizó la intervención.

Como resultado se encuentra el impacto de la implementación del programa denominado Grupos excepcionales y la estrategia pedagógica que fue positiva para los participantes, destacándose la apropiación de la metodología y los aprendizajes alcanzados durante las sesiones de taller. En este sentido, se puede definir que el programa contribuye a la apropiación de conductas resilientes y a fortalecer la importancia del rol del trabajador social en la intervención con este grupo. Sin embargo, se recomienda robustecer el programa mediante las competencias del trabajador social y el tipo de población atendida, permitiendo contar con una estrategia integral que reconozca las particularidades de los sujetos de intervención.

Es preciso señalar que el establecimiento penitenciario y carcelario cuenta con cinco patios donde la población privada de la libertad se encuentra organizada según los delitos y la extensión de la condena. Este grupo de personas que se encuentra recluida en el establecimiento está en un rango de edad entre los 18 y los 70 años.

Debe mencionarse también que en este centro carcelario los trabajadores sociales en formación intervienen en el área de atención y tratamiento, brindándoles a los internos atención psicosocial por medio de programas transversales que hacen parte de la intervención penitenciaria. A continuación, se mencionan algunos de ellos.

Grupos excepcionales (población discapacitada, persona mayor, afrodescendientes, indígenas y raizales): este programa busca la inclusión de estas personas bajo su particularidad e identificadas como población vulnerable. Allí se realizan acciones por medio del enfoque diferencial penitenciario,lo que disminuye la discriminación dentro del centro carcelario. Esta población está articulada al Programa de Intervención Penitenciaria para Adaptación Social (PIPAS) de condenados por delitos sexuales que busca reducir la presencia de factores de riesgo asociados a la reincidencia del comportamiento sexual delictivo. Además, está dirigido a hombres condenados por delitos sexuales y, dentro de su plan de tratamiento, pretende cumplir un objetivo terapéutico relacionado con la identificación de causas o factores asociados con el delito sexual mediante acciones de prevención frente al mismo.

En esta perspectiva, la evaluación del proceso a partir de la sistematización estuvo demarcada bajo un enfoque crítico. De acuerdo con Cifuentes (1999, p. 30), este es una posibilidad de análisis de las prácticas desde una mirada complejizadora que se fundamenta en una postura política transformadora que determina lineamientos propios de la inclusión en el contexto carcelario. Lo anterior se articula con la sistematización realizada en el INPEC de la ciudad de Girardot ya que se buscó reconocer las voces de quienes participan en la experiencia a partir de los lineamientos institucionales y participativos, dando lugar a resultados de la práctica que están sujetos a evaluación.

Dentro de esta postura crítica se encuentra la apuesta metodológica dialéctica en la que se enfatiza en el carácter reflexivo y de articulación entre teoría y práctica de la intervención profesional (promoción y educación). Lo anterior supone descartar los principios básicos del positivismo, pues el enfoque crítico implica comprometerse e interactuar con una intención política, cuestionar y reaccionar frente a las metodologías formales de las ciencias sociales. De paso, esta postura sitúa la metodología de sistematización de la práctica profesional frente a la identificación y evaluación de las acciones que promueven la formación o fomentan la conducta resiliente de la persona mayor del grupo excepcional en el INPEC. 
A su vez, la sistematización se fundamentó en un marco teórico concordante con las categorías de análisis propuestas. En primera instancia se postularon los aportes de Forés y Grané, quienes plantean los elementos básicos del desarrollo de la resiliencia. Así mismo,estos autores abordan aquellos factores protectores referidos a la comprensión de la fuente de la resiliencia y proponen una ruta de diez (10) caminos hacia la construcción de la misma a partir de la implementación de cambios en la forma de pensar y actuar. Ello se complementa con el concepto de sistemas relacionado con las implicaciones que tiene para el adulto mayor la familia resiliente, la resiliencia en la participación comunitaria y otras maneras de comprensión del concepto en las situaciones en que vive la persona, aplicables a la particularidad de la vida del adulto mayor en los centros penitenciarios y carcelarios (FORÉS \& GRANÉ, 2008, p. 146).

Otro referente importante son los aportes de Betancourt, Guevara y Fuentes (2011, p. 32-35), quien es identifican una metodología basada en talleres pedagógicos. Los autores mencionan que estos son una vía idónea para formar, desarrollar y perfeccionar hábitos, habilidades y capacidades que le permiten al individuo operar en el conocimiento, transformar el objeto y cambiarse a sí mismo. Los talleres pedagógicos se llevaron a cabo en cuatro momentos: identificación, apropiación, descubrimiento y, finalmente, el de las acciones, donde se realizan talleres centrados en la reflexión y planeación personal de conductas propias de la resiliencia aplicables a los adultos mayores que hacen parte del presente estudio (MAYA, 2007, p. 79).

Por último,debe destacarse que se fundamentaron los roles que cumple el trabajador social en la intervención social, especialmente en los ámbitos de desempeño en el área penitenciaria. Estos corresponden al papel de aquel como mediador, gestor, informador, comunicador, proveedor de servicios, facilitador, planificador y evaluador, para mejorar la calidad de vida de las personas privadas de la libertad. Este aspecto permitió fortalecer e implementar conductas resilientes en las personas privadas de la libertad (FERNÁNDEZ \& ALEMÁN, 2014, p. 306).

\section{Método y metodología}

Para realizar la sistematización es importante reconocer el contexto de la práctica profesional y analizar el resultado de la misma,de manera que se valore la mirada de los participantes (en este caso, las personas mayores privadas de la libertad del EPMS de Girardot) que hacen parte de la experiencia. Se destaca que este proceso centra su intervención enla población de adultos mayores, la cual se encuentra recluida en el Patio 5 de dicho establecimiento y pertenecen al programa de grupos excepcionales que brinda el área de atención y tratamiento cuyo objetivo es la resocialización de dicha población.

Los participantes para el presente estudio fueron veinte (20) personas mayores cuyas características particulares son las siguientes: nivel socioeconómico 1 y 2, rango de edad entre 62 y 71 años, delitos cometidos de abuso sexual y asociados con estupefacientes, tiempo de condena entre 4 y 17 años, lugar de procedencia variado (municipios como Agua de Dios, Melgar, Nilo, Coello y departamentos de Atlántico, Bolívar, Cesar, Tolima y Antioquia; incluso, se contó con la vinculación de un extranjero procedente de Perú) y nivel académico disímil (esto incluye personas sin escolaridad, algunas con secundaria incompleta y un profesional).

El método usado para desarrollar la sistematización se fundamenta en la investigación cualitativa, a partir del uso de técnicas e instrumentos como la entrevista, el sociodrama (colcha de retazos), los relatos de vida, los juegos de fotos y la ficha de recuperación de la experiencia. Los resultados y datos fueron analizados bajo el software de análisis cualitativo MaxQDA y NVivo 
11. Lo anterior facilitó nuevos aprendizajes frente a concepciones metodológicas y teóricas que se establecen con este tipo de instituciones y población. Así mismo, se asumió como punto de partida el enfoque crítico propuesto por Cadena (citado por CIFUENTES, 1999, p. 30), quien sugiere un esquema para sistematizar experiencias organizativas, de capacitación y promoción a partir de una serie fases, teniendo en cuenta que las mismas fueron desarrolladas durante el ejercicio de la práctica profesional del trabajador social. A renglón seguido se mencionan dichas fases.

Identificación de necesidades: en esta fase se describió cómo se realizó la práctica y quiénes participaron en la misma, definiendo actores en relación con profesionales que realizaron la intervención, actores directos e indirectos, protagonistas y los sujetos de intervención de la práctica. Formulación de objetivos: en ella se plantearon las metas a cumplir y la identificación de capacidades individuales y colectivas, teniendo en cuenta capacidades físicas, cognitivas y psicomotoras del grupo poblacional abordado. Por último, aquí también se identifica quién fue el responsable de la ejecución y el perfil de competencias con el que este cuenta.

Definición de estrategia metodológica: en esta fase se definió la modalidad pedagógica que se adoptó y se explica porqué se escogió, quién la llevó a cabo y cómo se vincula con actividades previas y posterior es tanto del proceso desarrollado como de otros programas.

Estructuración temática - Currículum: aquí se hizo una definición de contenidos temáticos en donde se articularon los temas propuestos. Además, se señala quién ejecutó la socialización de dichos temas y los criterios de selección de las temáticas desarrolladas.

Programación de la experiencia educativa: fase en la que se determinaron los momentos propicios del desarrollo de la temática. Así mismo, se identificó en qué medida se aprovecharon los recursos locales y quién desarrolló la programación.

Participantes: en esta fase se elaboró una caracterización de cada uno de los participantes, lo que permitió la construcción de un perfil socio económico, técnico y académico de los mismos. Así mismo, se establecieron algunos criterios adicionales en relación con la selección de los participantes, la invitación a los mismos y su ubicación en el programa de acción. Estas experiencias que han tenido frente a otros programas-eventos de formación de derechos humanos han permitido, finalmente, hacer un análisis de su desempeño en las capacitaciones y demás acciones de formación.

Facilitador o docente: aquí se describió el perfil del facilitador o docente, su grado de participación en las etapas anteriores, la interacción que tuvo con otros participantes, su selección y, finalmente,una descripción de su desempeño.

Material didáctico: en esta fase se enumeró y describió el material didáctico empleado; además, se identificó el personal que lo elaboró, la forma y la finalidad que se dio al uso del mismo.

Espacio educativo, equipo y otros materiales: en esta fase se abordaron aspectos relacionados con las características de los materiales, el equipo de trabajo y los recursos a usar en el proyecto (recurso humano, equipos, insumos y materiales); además, se especifica el aprovechamiento que se le da a cada uno de ellos.

Financiamiento: en esta fase se tuvo en cuenta el costo del proceso, los financiadores del mismo y la planeación del gasto.

Evaluación: la evaluación de la sistematización contó con dos escenarios: por una parte,el escenario ejercido por la trabajadora social de la institución en el que se revisó la estructura del programa, la pertinencia de las estrategias para el desarrollo del mismo y la intervención de los 
trabajadores sociales en formación. Por otro lado,el desarrollado por parte de la Universidad en donde se revisó la intervención y se realizó la valoración del documento final de sistematización de la experiencia.

Además de suplir cada uno de los ítems planteados en el mismo,la metodología favoreció la identificación de categorías de la sistematización y esclareció cada uno de los resultados en la intervención, así como el análisis de efectividad de la misma.

Al realizar la reconstrucción de la experiencia frente al fortalecimiento de la resiliencia en adultos mayores se pueden determinarlos momentos que a continuación se desglosan en el siguiente cuadro.

Cuadro 1. Momentos del proceso de formación

\begin{tabular}{|c|c|c|}
\hline Momento & Talleres / Responsables & Proceso de articulación \\
\hline \multirow{3}{*}{ 1. Identificación } & $\begin{array}{l}\text { Qué es resiliencia / } \\
\text { Trabajadores sociales en } \\
\text { formación. }\end{array}$ & $\begin{array}{l}\text { La actividad buscó la construcción del concepto de resiliencia, } \\
\text { con la premisa de "Crecer desde la adversidad". Con esta actividad } \\
\text { inicial se observó un conocimiento nulo del concepto, por lo que } \\
\text { se trató de un momento de construcción del mismo. }\end{array}$ \\
\hline & $\begin{array}{l}\text { Aspectos de la resiliencia } \\
\text { / Trabajadores sociales en } \\
\text { formación. }\end{array}$ & $\begin{array}{l}\text { Con el concepto definido se articuló el diagnóstico individual, } \\
\text { lo que permitió identificar a los participantes del grupo. En esta } \\
\text { actividad se usaron instrumentos propios del Trabajo Social de } \\
\text { Grupo. }\end{array}$ \\
\hline & $\begin{array}{l}\text { Fuentes de la resiliencia / } \\
\text { Trabajadores sociales en } \\
\text { formación. }\end{array}$ & $\begin{array}{l}\text { Se consideraron como fuentes para el trabajo cuatro ámbitos: } \\
\text { Yo tengo (apoyo social), Yo soy (fortaleza interna), Yo estoy } \\
\text { (dispuesto a hacer) y Yo puedo (habilidades). }\end{array}$ \\
\hline \multirow{3}{*}{ 2. Apropiación } & $\begin{array}{l}\text { Pilares de la resiliencia I / } \\
\text { Trabajadores sociales en } \\
\text { formación. }\end{array}$ & $\begin{array}{l}\text { Para el proceso de apropiación se partió de una serie de pilares } \\
\text { (capacidad de pensamiento crítico, confianza, autoestima, } \\
\text { introspección e independencia) con los que contaba la población } \\
\text { de adultos mayor es para el desarrollo de la resiliencia. }\end{array}$ \\
\hline & $\begin{array}{l}\text { Pilares de la resiliencia II } \\
\text { / Trabajadores sociales en } \\
\text { formación. }\end{array}$ & $\begin{array}{l}\text { La continuidad del trabajo se fundamentó sobre los siguientes } \\
\text { pilares: Ética, Capacidad de relacionarse, Iniciativa, Creatividad } \\
\text { y Humor en el contexto de la población objeto de intervención o } \\
\text { posterior sistematización. }\end{array}$ \\
\hline & $\begin{array}{l}\text { Construcción de la } \\
\text { resiliencia / Trabajadores } \\
\text { sociales en formación. }\end{array}$ & $\begin{array}{l}\text { Luego del trabajo con estos pilares se realizó el ejercicio de } \\
\text { construcción de la resiliencia, el cual permitió diagnosticar } \\
\text { recursos y potencialidades; tener en cuenta el entorno, la } \\
\text { persona (unidad) y la elección; integrar la experiencia pasada en } \\
\text { la vida presente;reivindicar la espontaneidad; reconocer el valor } \\
\text { de la imperfección; descubrir que el fracaso no anula el sentido; } \\
\text { adaptar la acción; imaginar nuevas formas y transformar su } \\
\text { contexto. }\end{array}$ \\
\hline
\end{tabular}


conclusão

\begin{tabular}{|c|c|c|}
\hline Momento & Talleres / Responsables & Proceso de articulación \\
\hline \multirow{3}{*}{ 3.Descubrimiento } & $\begin{array}{l}\text { Caminos para ser } \\
\text { resilientes / Trabajadores } \\
\text { sociales en formación. }\end{array}$ & $\begin{array}{l}\text { En este encuentro se definieron los siguientes caminos: visión } \\
\text { esperanzada de las cosas;mantener la perspectiva; alimentar } \\
\text { una visión positiva de uno mismo; actuar con decisión frente a } \\
\text { las adversidades; avanzar en forma realista hacia sus objetivos; } \\
\text { aceptar que los cambios son parte de la vida; evitar ver las } \\
\text { crisis como problemas insuperables; hacer conexiones y buscar } \\
\text { oportunidades para el autodescubrimiento. }\end{array}$ \\
\hline & $\begin{array}{l}\text { La resiliencia en la persona } \\
\text { mayor / Trabajadores } \\
\text { sociales en formación. }\end{array}$ & $\begin{array}{l}\text { Luego de esta definición de caminos se trabajó sobre los } \\
\text { aspectos que definen la resiliencia en las personas mayores. } \\
\text { Por tal motivo, se utilizó el diagnóstico para la construcción } \\
\text { personal y grupal. }\end{array}$ \\
\hline & $\begin{array}{l}\text { Enfoques de la resiliencia / } \\
\text { Trabajadores sociales en } \\
\text { formación. }\end{array}$ & $\begin{array}{l}\text { En este momento se trabajaron tres enfoques de la resiliencia: } \\
\text { uno basado en las experiencias, el segundo en la autoestima y } \\
\text { el tercero en el desarrollo de aptitudes y competencias en el } \\
\text { adulto mayor. }\end{array}$ \\
\hline \multirow{3}{*}{ 4. Acciones } & $\begin{array}{l}\text { Factores protectores de la } \\
\text { resiliencia / Trabajadores } \\
\text { sociales en formación. }\end{array}$ & $\begin{array}{l}\text { Se trabajaron acciones para la construcción de redes de } \\
\text { contactos para la aceptación básica de personas y la capacidad } \\
\text { para descubrir un sentido positivo hacia la vida por parte del } \\
\text { adulto mayor. }\end{array}$ \\
\hline & $\begin{array}{l}\text { Factores de riesgo de la no } \\
\text { resiliencia / Trabajadores } \\
\text { sociales en formación. }\end{array}$ & $\begin{array}{l}\text { De la mano con las acciones protectoras se realizaron talleres } \\
\text { para la comprensión de los riesgos evidentes que se generan por } \\
\text { no aceptar la resiliencia. }\end{array}$ \\
\hline & $\begin{array}{l}\text { Retroalimentación } \\
\text { grupal de la resiliencia / } \\
\text { Trabajadores sociales en } \\
\text { formación. }\end{array}$ & $\begin{array}{l}\text { Las actividades finales de retroalimentación permitieron } \\
\text { la articulación de los aprendizajes más significativos y las } \\
\text { consecuencias de la toma de decisiones de los adultos mayores } \\
\text { participantes en el marco de la construcción de la resiliencia. }\end{array}$ \\
\hline
\end{tabular}

Fuente: elaboración propia. 2018

Como resultado de la apropiación de procesos formativos, de promoción y educación a este grupo poblacional se describe un modelo pedagógico que no solo facilita la socialización de contenidos descritos en el primer y segundo momento, sino que fundamenta un ejercicio de reflexión y descubrimiento de aquellos momentos que han afectado su estado actual en el centro penitenciario, sus expectativas y su interés. Por último, valida las acciones que construyen y deconstruyen su actuar futuro respecto dela condena misma, facilitando futuras intervenciones o la continuidad de la misma.

\section{Resultados}

\section{Momentos históricos y experiencias}

Una vez reconstruido el proceso anterior frente a la sistematización y los momentos de la intervención con persona mayor se deducen los siguientes aspectos: 
Cuadro 2. Categorías, indicadores, recursos

\begin{tabular}{|c|c|c|c|c|c|c|}
\hline Categoría & Objetivo & Actividades & indicadores & Instrumentos & Recursos & Evidencias \\
\hline $\begin{array}{l}\text { Estrategias } \\
\text { pedagógicas }\end{array}$ & $\begin{array}{l}\text { Determinar si } \\
\text { la estrategia } \\
\text { pedagógica } \\
\text { desarrollada por } \\
\text { las trabajadoras } \\
\text { sociales en } \\
\text { formación fue } \\
\text { acorde con el } \\
\text { proceso. }\end{array}$ & \begin{tabular}{|l} 
Diseño, \\
implementación \\
y puesta en \\
marcha de \\
los talleres \\
educativos \\
durante el \\
periodo de 2017.
\end{tabular} & $\begin{array}{l}\text { Realización } \\
\text { de doce (12) } \\
\text { talleres donde se } \\
\text { trataron temas } \\
\text { fundamentales } \\
\text { en relación con la } \\
\text { resiliencia. }\end{array}$ & $\begin{array}{l}\text { Diseño de plan } \\
\text { de cada una } \\
\text { de las sesiones } \\
\text { de los talleres } \\
\text { implementados } \\
\text { en la población } \\
\text { de adulto } \\
\text { mayor. }\end{array}$ & $\begin{array}{l}\text { Recursos físicos: } \\
\text { planta física, } \\
\text { marcadores, } \\
\text { cartulinas, } \\
\text { colores. }\end{array}$ & $\begin{array}{l}\text { Registro de } \\
\text { asistencia y } \\
\text { fotografías delas } \\
\text { actividades. }\end{array}$ \\
\hline $\begin{array}{l}\text { Conducta } \\
\text { resiliente }\end{array}$ & $\begin{array}{l}\text { Establecer la } \\
\text { relación entre } \\
\text { las conductas } \\
\text { resilientes y la } \\
\text { exclusión social } \\
\text { sufrida por } \\
\text { las personas } \\
\text { mayores en el } \\
\text { EPMSC. }\end{array}$ & $\begin{array}{l}\text { Evaluación de } \\
\text { los talleres con } \\
\text { las personas } \\
\text { mayores acerca } \\
\text { de las conductas } \\
\text { resilientes. }\end{array}$ & $\begin{array}{l}\text { Número de } \\
\text { personas que } \\
\text { consideran } \\
\text { que a través } \\
\text { de los talleres } \\
\text { adquirieron } \\
\text { capacidades para } \\
\text { una conducta } \\
\text { resiliente. }\end{array}$ & $\begin{array}{l}\text { Encuestas y } \\
\text { evaluación } \\
\text { aplicada a los } \\
\text { participantes } \\
\text { al final de cada } \\
\text { taller. }\end{array}$ & $\begin{array}{l}\text { Lápices, hojas y } \\
\text { computador. }\end{array}$ & $\begin{array}{l}\text { Evaluaciones y } \\
\text { tabulación de } \\
\text { las encuestas } \\
\text { aplicadas a los } \\
\text { participantes. }\end{array}$ \\
\hline $\begin{array}{l}\text { Rol del } \\
\text { trabajador } \\
\text { social }\end{array}$ & $\begin{array}{l}\text { Determinar si } \\
\text { los aspectos } \\
\text { metodológicos y } \\
\text { procedimentales } \\
\text { del quehacer } \\
\text { del trabajador } \\
\text { social generaron } \\
\text { conductas } \\
\text { resilientes para } \\
\text { las personas } \\
\text { mayores } \\
\text { privadas de la } \\
\text { libertad. }\end{array}$ & $\begin{array}{l}\text { Evaluación de } \\
\text { la intervención } \\
\text { profesional de } \\
\text { los trabajadores } \\
\text { sociales del } \\
\text { programa. }\end{array}$ & $\begin{array}{l}\text { Número de } \\
\text { personas } \\
\text { atendidas a través } \\
\text { de los talleres } \\
\text { formulados desde } \\
\text { el Área de Trabajo } \\
\text { Social para la } \\
\text { persona mayor en } \\
\text { la búsqueda del } \\
\text { fortalecimiento } \\
\text { de conductas } \\
\text { resilientes. }\end{array}$ & $\begin{array}{l}\text { Evaluación de } \\
\text { la construcción, } \\
\text { el diseño y la } \\
\text { implementación } \\
\text { de los talleres } \\
\text { desarrollados } \\
\text { por los } \\
\text { trabajadores } \\
\text { sociales. }\end{array}$ & $\begin{array}{l}\text { Trabajadores } \\
\text { sociales en } \\
\text { formación e } \\
\text { interlocutores. }\end{array}$ & $\begin{array}{l}\text { Instrumento } \\
\text { de evaluación; } \\
\text { encuesta aplicada } \\
\text { a los adultos } \\
\text { mayores. }\end{array}$ \\
\hline
\end{tabular}

Fuente: elaboración propia. 2018

Las categorías seleccionadas se articulan con aspectos propios de la intervención profesional, las teorías utilizadas y el eje orientador de la sistematización. Lo anterior representa la información que se indagó y analizó para que el quehacer profesional en Trabajo social en los centros penitenciarios se articule con las dependencias que adelantan acciones con los internos encaminados a generar su resocialización por medio de diferentes actividades y procesos. Así, la sistematización de la intervención en Trabajo Social estuvo adscrita al área denominada Atención y tratamiento, cuya misión es contribuir al desarrollo y a la resignificación de las potencialidades de las personas privadas de la libertad a través de los servicios de tratamiento penitenciario, atención básica y seguridad, cimentados en el respeto de los derechos humanos y el fomento de la gestión ética y transparente.

Para la puesta en marcha de los distintos programas el área de atención y tratamiento realizó entrevistas y evaluaciones a cada interno. La intención con esto fue verificar que cumplieran con las características y condiciones requeridas para el ingreso a los mismos, de manera que se pudiera comenzar con el tratamiento penitenciario. Estas entrevistas fueron analizadas mediante el software MaxQDA. 
Figura 1. Mapa de nube

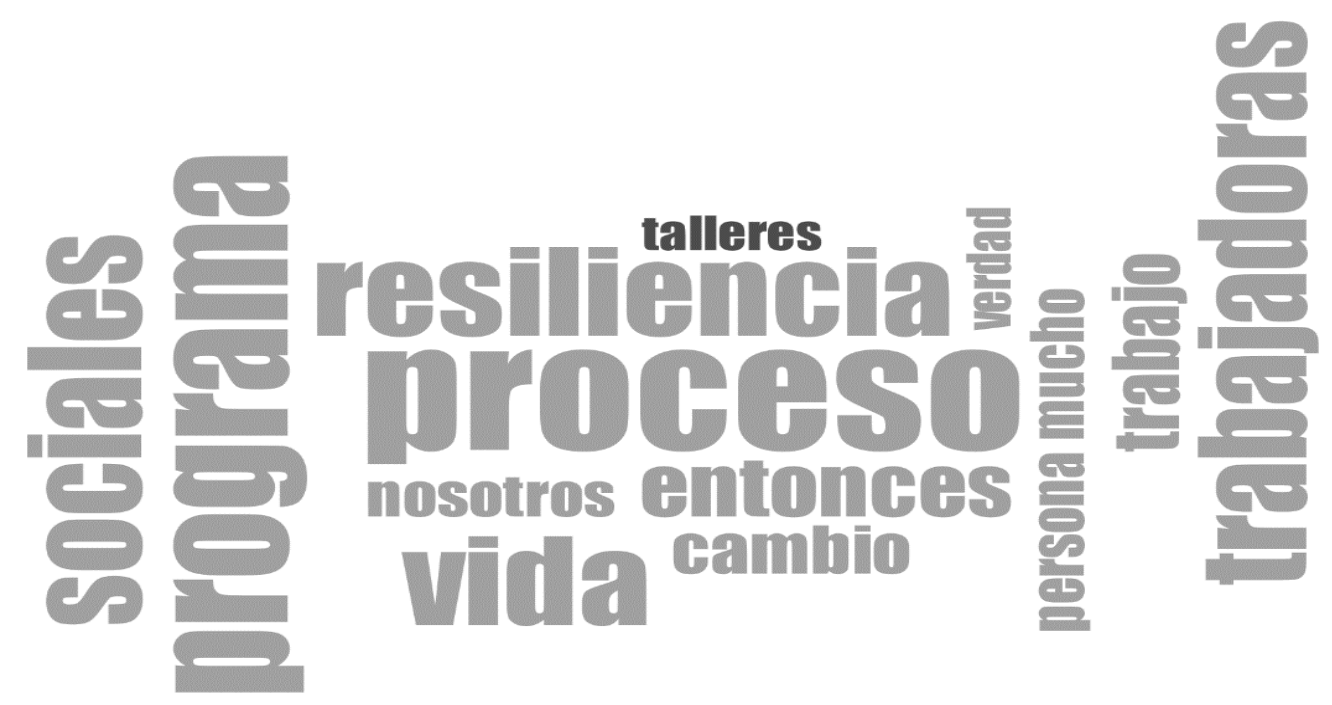

Fuente: elaboración propia MaxQDA. 2018

Al realizar el conteo de palabras descritas en las entrevistas y analizadas bajo el software mencionado, se evidencia que la consideración de la resiliencia se presentó como una descripción por parte de los participantes. Lo anterior resulta normal si se entiende que se hizo énfasis en la participación de los talleres en el marco del programa. De igual manera, se destacan las palabras vida, proceso y resiliencia, las cuales hacen parte fundamental del lenguaje del programa y los contenidos temáticos. Por ello se puede validar, desde el mapa de nube, una reflexión valorativa de los conceptos clave y temas tratados, dándose le especial importancia a los programas del INPEC, a las practicantes y a aspectos generales de la resiliencia.

En el libro Crecer desde la adversidad (FORÉS \& GRANÉ, 2008, p. 12) se identifican temáticas importantes para la resiliencia y se propone la ruta de la resiliencia activa, que nace de la comprensión de los aprendizajes de la experiencia de aplicación del programa en el centro penitenciario de Girardot. El objetivo de la ruta es establecer una metodología para la generación de conductas resilientes en grupos de adultos mayores centrada en personas privadas de la libertad. Sin embargo, como se observa en el diseño, esta ruta puede ser incorporada a diferentes estrategias aplicadas a individuos privados de la libertado con adultos mayores que se encuentren en otros contextos o condiciones.

Esta ruta de resiliencia se reconoce como una herramienta que permite un mejoramiento delas condiciones de vida de las personas. De hecho, su aplicación puede darse en diferentes contextos, en especial en todos aquellos donde las personas necesiten restablecer su confianza, sus valores y realizar un autorreconocimiento siempre que estén afectadas por situaciones extremas que hayan motivado la privación de la libertad. Lo anterior se hace partiendo dela generación de una metodología propia del Modelo humanista-existencial, el cual propone una concepción optimista del ser humano que se genera desde su capacidad de cambio y aptitud de trascender hacia estados de mejora que permitan transformar sus problemas. Este modelo se fundamenta en la conciencia de sí mismo,reivindica la identificación de los determinismos sociales y personales que provocan la pérdida de sentido y las afectaciones en la calidad de vida.

Por otra parte, como propuesta de mejoramiento de la intervención profesional se propone la siguiente ruta de atención. Esta se encuentra particularmente dirigida a todos los grupos de 
personas (transversal) que hacen parte de la población privada de la libertad a partir de lo que a continuación se menciona.

\section{Aprendizajes - Ruta de la resiliencia activa}

La siguiente ruta tuvo como soporte metodológico la intervención social con grupos, reconocida como un método propio de intervención de la disciplina de Trabajo Social (GNNECO, 2005, p. 57). Define el Trabajo Social de Grupo como un método que fomenta el desempeño social de las personas a través de experiencias grupales, con el fin de desarrollar en los sujetos potencialidades y mejorar las relaciones entre ellos.

Dentro de este método existen diferentes tipos de grupos a intervenir, en los que se debe tener presente el objetivo de la intervención, las necesidades e intereses de la misma. Así, la población objeto de intervención del programa descrito se enmarca como un grupo de resocialización, con el que se tiene como objetivo ayudar a los miembros a cambiar su conducta-considerada como desviada-y mejorar su desempeño social.

En este orden de ideas, la ruta de la resiliencia activa se desarrolla desde acciones determinantes como la promoción de actividades culturales, la asistencia en servicios sociales, el mejoramiento de condiciones económicas, de ambientes físicos y sociales, entre otros aspectos. Se puede deducir que el trabajo sobre resiliencia se puede leer como una oportunidad de crecimiento importante para el mejoramiento de la calidad de vida de las personas mayores.

Cuadro 3. Aprendizaje y ruta

\begin{tabular}{|l|l|l|l|l|}
\hline Temáticas & Contenidos & Objetivo & Metodología & Recursos \\
\hline Resiliencia & $\begin{array}{l}\text { Concepto de } \\
\text { resiliencia. }\end{array}$ & $\begin{array}{l}\text { Lograr que la } \\
\text { persona mayor } \\
\text { privada de la } \\
\text { libertad tenga } \\
\text { un acercamiento } \\
\text { al concepto de } \\
\text { resiliencia y la } \\
\text { importancia de la } \\
\text { misma. }\end{array}$ & $\begin{array}{l}\text { Proyección de la película El circo de la } \\
\text { mariposa. De forma creativa (mediante } \\
\text { escritos, dibujos y dramatizados) se dio a } \\
\text { conocer lo que sintieron los reclusos con } \\
\text { esta película y se les solicitó realizar una } \\
\text { asociación con situaciones de sus vidas. }\end{array}$ & $\begin{array}{l}\text { Proyección visual } \\
\text { de la } \\
\text { película, } \\
\text { hojaces, colores y } \\
\text { temperas. }\end{array}$ \\
\hline $\begin{array}{l}\text { Fuentes de la } \\
\text { resiliencia }\end{array}$ & $\begin{array}{l}\text { Yo tengo (apoyo } \\
\text { social), } \\
\text { Yo soy (fortaleza } \\
\text { interna), } \\
\text { Yo estoy (dispuesto a } \\
\text { hacer), } \\
\text { Yo puedo } \\
\text { (habilidades). }\end{array}$ & $\begin{array}{l}\text { Desarrollar } \\
\text { autoconocimiento } \\
\text { de las habilidades } \\
\text { yotencialidades } \\
\text { resilientes que } \\
\text { poseen los } \\
\text { participantes. }\end{array}$ & $\begin{array}{l}\text { Se realizó un mural de resiliencia donde } \\
\text { los participantes pudieron plasmar el } \\
\text { apoyo social con el que cuentan, las } \\
\text { fortalezas internas que los llevan a hacer } \\
\text { cada día transformaciones en su vida, lo } \\
\text { que están dispuestos a hacer para cambiar } \\
\text { lo negativo por lo positivo y la forma } \\
\text { como esto se transforma en habilidades } \\
\text { resilientes (ver Figura 2). }\end{array}$ & $\begin{array}{l}\text { Papel kraft, } \\
\text { temperas, } \\
\text { pinceles, } \\
\text { cinta. }\end{array}$ \\
\hline
\end{tabular}


continuação

\begin{tabular}{|c|c|c|c|c|}
\hline Temáticas & Contenidos & Objetivo & Metodología & Recursos \\
\hline $\begin{array}{l}\text { Aspectos de la } \\
\text { resiliencia }\end{array}$ & $\begin{array}{l}\text { Amor, experiencias, } \\
\text { autoestima, aptitudes } \\
\text { y competencias, } \\
\text { humor, capacidad de } \\
\text { construir un sentido, } \\
\text { redes de contactos y } \\
\text { aceptación básica de } \\
\text { personas. }\end{array}$ & $\begin{array}{l}\text { Ampliar la } \\
\text { importancia de } \\
\text { los valores y de } \\
\text { las habilidades en } \\
\text { la persona mayor } \\
\text { fortaleciendo la } \\
\text { resiliencia. }\end{array}$ & $\begin{array}{l}\text { Para esto se creó La casita de la resiliencia, } \\
\text { propuesta por Stefan Vanistendael y } \\
\text { Jaques Lecomte (como se citó en MUÑOZ } \\
\text { \& DE PEDRO, 2005, p. 107). } \\
\text { Esta casa permite simplificar la vivencia y } \\
\text { la compresión para ver cómo la resiliencia } \\
\text { influye en la trasformación del individuo. } \\
\text { Primero se construyó el suelo que } \\
\text { constituye las necesidades básicas } \\
\text { (vivienda, alimento, ropa); luego el } \\
\text { cimiento,que responde a la confianza } \\
\text { adquirida gracias al vínculo que nos une } \\
\text { a la familia, a los amigos y al hecho de } \\
\text { sentirnos aceptados y comprendidos; } \\
\text { subiendo al primer piso, en este se sitúa la } \\
\text { búsqueda de sentido, el por qué y el para } \\
\text { qué de las cosas. } \\
\text { En el segundo piso se sitúan elementos } \\
\text { como las aptitudes personales y sociales, } \\
\text { la autoestima e incluso el sentido del } \\
\text { humor. } \\
\text { Por último,debe mencionarse el } \\
\text { techo donde se da apertura a nuevas } \\
\text { experiencias y elementos que ayudan a la } \\
\text { construcción de la resiliencia. }\end{array}$ & $\begin{array}{l}\text { Hojas de block, } \\
\text { papel iris, } \\
\text { lápices, } \\
\text { colores y } \\
\text { marcadores. } \\
\end{array}$ \\
\hline $\begin{array}{l}\text { Construcción } \\
\text { de la } \\
\text { resiliencia }\end{array}$ & $\begin{array}{l}\text { Diagnosticar recursos } \\
\text { y potencialidades } \\
\text { teniendo en cuenta el } \\
\text { entorno. } \\
\text { Integrar experiencias } \\
\text { pasadas en la vida } \\
\text { presente. } \\
\text { Reconocer el valor de } \\
\text { la imperfección. } \\
\text { Considerar que el } \\
\text { fracaso no anula el } \\
\text { sentido. } \\
\text { Generar capacidades } \\
\text { para transformar su } \\
\text { contexto. }\end{array}$ & $\begin{array}{l}\text { Construir la } \\
\text { resiliencia mediante } \\
\text { las familias de las } \\
\text { personas mayores, } \\
\text { teniendo en cuenta } \\
\text { la importancia } \\
\text { de las mismas en } \\
\text { el proceso de ser } \\
\text { sujetos resilientes. }\end{array}$ & $\begin{array}{l}\text { Se realizó una cartografía familiar, } \\
\text { herramienta que permite conocer la } \\
\text { realidad de la vida cotidiana y reconocer } \\
\text { los espacios donde se genera mayor } \\
\text { afinidad y conflicto. Es por esto que la } \\
\text { cartografía permite construir memorias } \\
\text { para indagar sobre lo que estas atraviesan } \\
\text { en relación con la herencia, las relaciones } \\
\text { de género, las relaciones de poder, entre } \\
\text { otras variables (SANTACRUZ, 2016, p. 34). } \\
\text { Para esto se les pidió a los participantes } \\
\text { del grupo que realizaran una maqueta } \\
\text { de su casa e identificaran cuáles son los } \\
\text { lugares de la casa donde se generan las } \\
\text { siguientes situaciones: } \\
\text { 1.Discusiones } \\
\text { 2.Diálogos en familia } \\
\text { 3.Reconciliaciones } \\
\text { 4.Toma de decisiones }\end{array}$ & $\begin{array}{l}\text { Cartulina, } \\
\text { foami, } \\
\text { temperas, } \\
\text { cinta, } \\
\text { pegante, } \\
\text { marcadores y } \\
\text { lápices. }\end{array}$ \\
\hline
\end{tabular}


conclusão

\begin{tabular}{|c|c|c|c|c|}
\hline Temáticas & Contenidos & Objetivo & Metodología & Recursos \\
\hline $\begin{array}{l}\text { Pilares de la } \\
\text { resiliencia }\end{array}$ & $\begin{array}{l}\text { Humor, creatividad, } \\
\text { iniciativa, capacidad } \\
\text { de relacionarse, } \\
\text { ética, independencia, } \\
\text { introspección, } \\
\text { autoestima, confianza } \\
\text { y capacidad de } \\
\text { pensamiento crítico. }\end{array}$ & $\begin{array}{l}\text { Realizar un } \\
\text { acercamiento a } \\
\text { los pilares de la } \\
\text { resiliencia con el fin } \\
\text { de que la persona } \\
\text { mayor conozca } \\
\text { su significado y } \\
\text { la importancia de } \\
\text { hacer uso de ellos } \\
\text { en su diario vivir. }\end{array}$ & $\begin{array}{l}\text { Se socializaron cada uno de los pilares de } \\
\text { la resiliencia por medio de diapositivas. } \\
\text { Luego de haber hecho un acercamiento a } \\
\text { cada uno de los pilares se crearon grupos } \\
\text { de tres personas. En estos se representa } \\
\text { roncada uno de los pilares en forma de } \\
\text { sociodrama o mediante juegos de roles, } \\
\text { asociándolos con experiencias de la } \\
\text { vida por dentro o por fuera del centro } \\
\text { penitenciario. } \\
\text { Esto permitió hacer una reflexión acerca } \\
\text { de los momentos de la vida en que } \\
\text { pudieron o podrán poner en práctica } \\
\text { dicho tema. }\end{array}$ & $\begin{array}{l}\text { Proyección } \\
\text { visual para la } \\
\text { socialización de los } \\
\text { temas. }\end{array}$ \\
\hline $\begin{array}{l}\text { Caminos de la } \\
\text { resiliencia }\end{array}$ & \begin{tabular}{|l|} 
Buscar oportunidades \\
para el \\
autodescubrimiento. \\
Generar redes de \\
apoyo. \\
Evitar ver las crisis \\
como problemas \\
insuperables. \\
Aceptar que los \\
problemas hacen \\
parte de la vida. \\
Actuar con decisión \\
ante la adversidad. \\
Alimentar una visión \\
positiva de uno \\
mismo.
\end{tabular} & $\begin{array}{l}\text { Conocer los } \\
\text { diferentes caminos } \\
\text { que ayudan a la } \\
\text { persona mayor a ser } \\
\text { un sujeto resiliente } \\
\text { frente a las } \\
\text { adversidades que se } \\
\text { les presentan. }\end{array}$ & $\begin{array}{l}\text { Se realizó un árbol de manera individual } \\
\text { en el que se incluyeron tanto los factores } \\
\text { protectores como los de riesgo que se } \\
\text { presentan en el centro penitenciario. Así } \\
\text { mismo, se identificaron las relaciones } \\
\text { externas con la familia y los amigos en la } \\
\text { búsqueda de ser sujetos resilientes. } \\
\text { Este árbol se conformó de la siguiente } \\
\text { forma: en el tallo, las personas con las que } \\
\text { cuentan (familia, amigos y compañeros } \\
\text { del centro penitenciario); en las ramas, los } \\
\text { factores protectores que estos le ofrecen; } \\
\text { y en las hojas los factores de riesgo. } \\
\text { Para esto se socializó previamente lo que } \\
\text { se entiende por factores de riesgo y lo que } \\
\text { se asume por factores protectores. }\end{array}$ & $\begin{array}{l}\text { Papel kraft } \\
\text { lápices, } \\
\text { colores, } \\
\text { marcadores, } \\
\text { cinta y } \\
\text { proyección visual. }\end{array}$ \\
\hline
\end{tabular}

Fuente: elaboración propia. 2018

El objetivo de la ruta es establecer una metodología para la generación de conductas resilientes en grupos de adultos mayores. Pese a que dicha metodología está centrada en personas privadas de la libertad, como se observa en el proceso de diseño, esta ruta puede ser incorporada en la atención bien sea con personas privadas de la libertad, ocon adultos mayores en otros contextos. 
Figura 2. Mural de resiliencia.

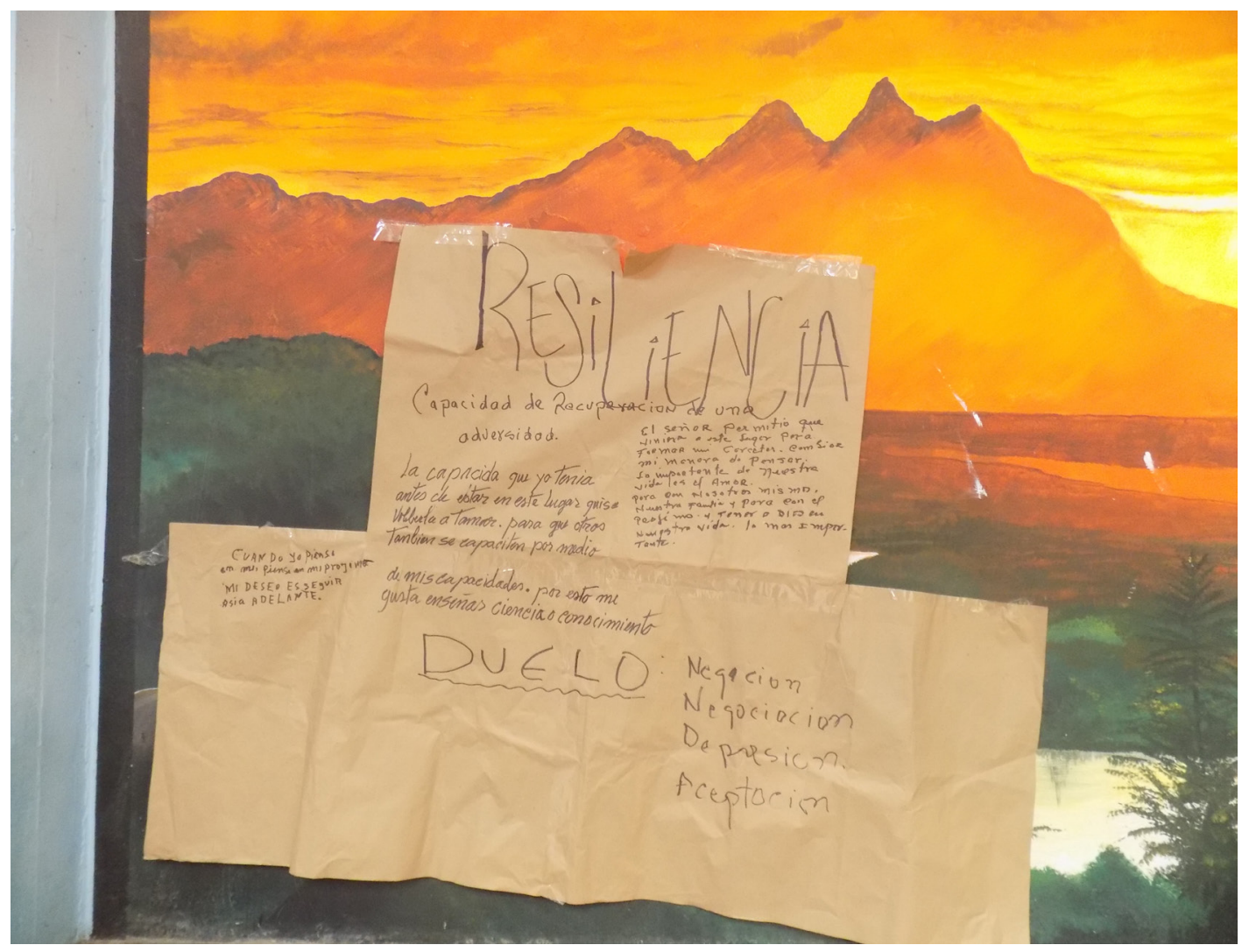

Fuente: fotografía tomada de la actividad Fuentes de la resiliencia. Elaboración propia.

\section{Conclusiones}

Luego de la sistematización es posible concluir que fue importante la presencia del trabajador social en los centros penitenciarios y que la intervención transformó las maneras de pensar, actuar y la forma de ver la vida de los participantes. Del mismo modo, la población -las personas mayores del centro penitenciario-es muy vulnerable y sufre de exclusión social debido a su condición y en razón a la percepción que genera el delito cometido. Por esto resulta significativa la intervención con este tipo de programas.

A esto se suma que la vejez se asume como una situación de vulnerabilidad en la que los adultos mayores son objeto de todo tipo de afectaciones sociales, individuales, de salud física y mental. Esto se materializa en síntomas de depresión, angustia, baja autoestima, entre otros que afectan de manera significativa la calidad de vida del adulto mayor y que deben ser comprendidos como una problemática seria de salud pública que requiere la intervención de profesionales en la materia. Por esto se plantea la necesidad de que surjan iniciativas desde la academia y desde las organizaciones del Estado que permitan mejorar las condiciones de envejecimiento del adulto mayor en Colombia.

Por tal motivo, esta población requiere de un constante acompañamiento que le permita una mejor convivencia y la superación de todas las situaciones que afectan su calidad de vida. En este sentido, es de destacar el impacto que tienen las estrategias pedagógicas desarrolladas, un aporte significativo y una herramienta valiosa para el trabajador social en sus labores profesionales. 
De igual manera, se destaca la importancia de la construcción de conductas resilientes en la población privada de la libertad. De hecho, a la luz de la teoría y con la aplicación de estrategias se superan las adversidades de la vida y descubren habilidades, potencialidades y acciones concretas que contribuyen a mejorar la calidad de vida de estas personas, potencializando sus habilidades y redescubriendo el sentido de la vida en las dimensiones personal y social. Así se puede establecer que este tipo de programas fomentan la prevención de nuevas conductas delictivas por abuso sexual.

Por último, es valioso sistematizar la experiencia de intervención, ya que es enriquecedora y pertinente para los trabajadores sociales en formación. Desde allí es posible evaluar el impacto delas diferentes estrategias que se utilizaron dentro de la aplicación sistemática de los conocimientos y el desarrollo de sus competencias profesionales, desde donde se toma lo bueno para perfeccionar lo y lo malo para replantearlo o cambiarlo.

\section{Referencias}

BETANCOURT, R.; GUEVARA, L. y FUENTES, E. El taller como estrategia didáctica, sus fases y componentes para el desarrollo de un proceso de cualificación en el uso de tecnologías de la información y la comunicación (tic) con docentes de lenguas extranjeras. Caracterización y retos. Repositorio de la Universidad de la Salle. 2011. Recuperado el 3 de Julio de 2019, de http://repository. lasalle.edu.co/bitstream/handle/10185/7927/T26.11\%20B465f.pdf

CADENA, F. La Sistematización como proceso. Revista Aportes, 32, p. 41-52, 1987.

CIFUENTES, R. La Sistematización de la Práctica del Trabajo Social. Argentina: Lumen \& Hvmanitas, 1999.

FERNÁNDEZ, T. y ALEMÁN, C. Introducción al Trabajo Social. Madrid, España: Alianza Editorial, 2014.

FORÉS, A. y GRANÉ, J. La Resiliencia. Crecer desde la Adversidad. Vol. 1. Barcelona, España: Plataforma Editorial, 2008.

GNNECO, M. Trabajo social con grupos fundamentos y tendencias. Bogotá: Kimpress, 2005.

GUZMÁN, J. Envejecimiento y desarrollo en América Latina y el Caribe. 2002. Recuperado el 9 de julio de 2019, de https://repositorio.cepal.org/bitstream/handle/11362/7166/1/S025388_es.pdf

MAYA, A. El Taller Educativo. Vol. 2. Bogotá: Cooperativa Editorial Magisterio. 2007.

MAYA, A. Pedagogía de la ternura. 2001. Recuperado el 9 de julio de 2019,de https://docplayer. es/35685584-Arnobio-maya-betancourt.html

MUÑOZ, V. y DE PEDRO, F. Educar para la resiliencia. Una cambio de mirada en la prevención de situaciones de riesgo social. Revista Complutense de Educación, 16(1), p. 107-124, 2005.

SANTACRUZ, N. Cartografía de prácticas familiares. Construcción de vínculos y relaciones. 2016. Recuperado el 9 de julio de 2019, de http://repositorio.puce.edu.ec/handle/22000/12216 